\title{
Quality Evaluation of Walnut Oil through HPLC and in Vitro Antioxidant Activity
}

\author{
Xu Li ${ }^{1,2}$, Yang Zhao ${ }^{1,2}$, Xiaojian Gong ${ }^{1,2}$, Chao Zhao ${ }^{1,2, *}$, Xin Zhou ${ }^{1,2, *}$ \\ ${ }^{1}$ Guizhou Mountain Environmental information System and Ecological Environmental Protection Key Laboratory, Guizhou Normal \\ University, Guiyang City, Guizhou Province, P. R. of China \\ ${ }^{2}$ The Research Center for Quality Control of Natural Medicine, Guizhou Normal University, Guiyang City, Guizhou Province, P. R. of \\ China \\ *Corresponding author: chaozhao@126.com, alice9800@sina.com
}

Received May 12, 2014; Revised May 21, 2014; Accepted May 23, 2014

\begin{abstract}
In the present study, a highly sensitive and simple high-performance liquid chromatographic method was developed and validated for simultaneous determination of three unsaturated fatty acids, $\alpha$ - linolenic acid, linoleic acid and oleic acid in walnut oil. Unsaturated fatty acids were quantified through HPLC using a reversephase C8 column and UV detector. Besides, the antioxidant activity of walnut was measured through in vitro tests including DPPH, ABTS and FRAP assay. Results showed that $\alpha$ - linolenic acid, linoleic acid and oleic acid ranged from 7.90 to $13.64,47.10$ to $56.58,11.81$ to $27.27 \mathrm{~g} / 100 \mathrm{~g}$ of walnut oil. The walnut oil exhibited antioxidant activity.
\end{abstract}

Keywords: walnut oil, HPLC, unsaturated fatty acids, quantification, antioxidant activities

Cite This Article: Xu Li, Yang Zhao, Xiaojian Gong, Chao Zhao, and Xin Zhou, "Quality Evaluation of Walnut Oil through HPLC and in Vitro Antioxidant Activity.” Journal of Food and Nutrition Research, vol. 2, no. 5 (2014): 244-249. doi: 10.12691/jfnr-2-5-6.

\section{Introduction}

Walnut (Juglans regia L.) is a highly nutritious food, which is rich in polyunsaturated fatty acids (PUFAs) and has shown to protect the brain from numerous insults associated with aging and may possibly allay age-related cognitive decline [10]. Walnut kernels generally contain about $60 \%$ oil [26], but this can vary from 52 to $70 \%$ depending on the cultivar, location grown, and irrigation rate $[6,16,18]$. The major constituents of the oil are triacylglycerols; free fatty acids, diacylglycerols, monoacylglycerols, sterols, sterol esters, and phosphatides are all present in only minor quantities [26]. The major fatty acids found in walnut oil are oleic (18:1), linoleic (18:2), and linolenic (18:3) acids. According to Simopoulos, walnuts are unique because they have a perfect balance of n-6: n-3 PUFA, a ratio of $4: 1$, which was showed to decrease the incidence of cardiovascular risk [2,5]. So, it's necessary to establish a simple, rapid simultaneous determination of unsaturated fatty acids content in the walnut oil. The major fatty acids in walnut oil were determined by GC or GC - MS usage. Gas chromatography is often derivative preprocessing, and the system is easy to make high temperature of $\alpha$ linolenic acid, linoleic acid and oleic acid fracture or double bond isomerization [1,11].

Using HPLC-UV method can also determine the fatty acids in oils and fats, but more work needs to be derived, sample pretreatment, long analysis time and influencing factors of many faults $[14,32]$. The present study uses the walnut oil saponification acid solution without derivatization process, established a simultaneous determination of $\alpha$ - linolenic acid, linoleic acid and oleic acid analysis method, and evaluated antioxidant activities of walnut oil through in vitro tests including $\mathrm{DPPH}$, ABTS and FRAP assay.

\section{Experimental}

\subsection{Chemicals, Reagents and Materials}

Acetonitrile used for HPLC was purchased from TEDIA (USA). The water was purified with a Milli-Q system. Ethanol and n-hexane of analytical grade were purchased from Bo Di Chemical Reagent Factory, Tian Jin, China. The $\alpha$-linolenic acid, linoleic acid and oleic acid of purity greater than $98 \%$ were purchased from Dr.Ehrenstorfer LTD (Made in Germany). 2,2-Diphenyl1-picrylhydrazyl (DPPH), 6-hydroxy-2,5,7,8tetramethylchroman-2-carboxylic acid (Trolox), 2,2' azinobis (3-ethylbenzothiazoline-6-sulfonic acid) diammonium salt (ABTS) and 2,4,6-tri(2-pyridyl)-striazine (TPTZ) were purchased from Sigma-Aldrich (France). The rest of reagents were of analytical grade. The walnuts were purchased on the local market and were maintained in the dark, at room temperature until analyses.

\subsection{Preparation of the Oil Sample and Walnut Extract}

The procedure described by Tian et al. [28] was used for the extraction of with some modifications. Before each kind of analysis the walnuts were manually cracked and 
shelled, and then chopped in an appliance mill. The crude oil was obtained from finely chopped nuts (ca.5g) reflux extracted with $10 \mathrm{~mL}$-hexane at $40^{\circ} \mathrm{C}$ for $1 \mathrm{~h}$. The extract solution was centrifuged at $3,000 \mathrm{~g}$ for $10 \mathrm{~min}$. Take on the supernatant, the remaining solvent was removed by vacuum distillation. $100 \mathrm{mg}$ of the walnut oil was dissolved in $1 \mathrm{~mL}$ isopropanol. An aqueous-organic extraction was performed in walnut kernel, by placing $0.5 \mathrm{~g}$ of sample in a conical flask and adding $20 \mathrm{~mL}$ of $60 \%$ ethanol at $49^{\circ} \mathrm{C}$ for $1 \mathrm{~h}$. The extract solution was centrifuged at 3,000g for $10 \mathrm{~min}$ and the supernatant was recovered, then the walnut kernel extract was kept at $-20^{\circ} \mathrm{C}$.

\subsection{Preparation of the Standard and Fatty Acid Sample Solutions}

The $\alpha$-linolenic acid, linoleic acid and oleic acid were accurately weighed and dissolved in nonaqueous ethanol at $282.8 \mathrm{ug} / \mathrm{mL}, 154.5 \mathrm{ug} / \mathrm{mL}$ and $255.8 \mathrm{ug} / \mathrm{mL}$. To $0.5 \mathrm{~g}$ of the crude oil added $5 \mathrm{~mL}$ of $1 \mathrm{~mol} / \mathrm{L}$ sodium hydroxideethanol solution, then saponified at $70^{\circ} \mathrm{C}$ for $1 \mathrm{~h}$. When the saponification solution cool, joined the $20 \%$ hydrochloric acid to pH3-4. The solution was transferred into $25 \mathrm{~mL}$ volumetric flask, washed the conical flask with ethanol, and transferred the liquid detergent into $25 \mathrm{~mL}$ volumetric flask, diluted to $25 \mathrm{~mL}$. Then, accurately transferred $100 \mathrm{uL}$ sample solution to $2 \mathrm{~mL}$ volumetric flask and diluted to $2 \mathrm{~mL}$ with nonaqueous ethanol. The sample solution was filtered through a $0.45 \mu \mathrm{m}$ membrane prior to an injection into HPLC system. The contents of the analytes were determined from the corresponding calibration curves [35].

\subsection{HPLC Analysis}

All separations were performed on a Dionex Ultimate 3000 liquid chromatograph, equipped with a degasser, a quaternary pump, an autosampler, and an UV detector. The date was acquired and processed using Chromeleon 7.1 ChemStation software. Chromatography was carried out on a ZORBAX Eclipse XDB-C8 column $(4.6 \times 150 \mathrm{~mm}$, $5 \mu \mathrm{m})$ at a column temperature of $30^{\circ} \mathrm{C}$.

The optimum resolution was obtained by using an isocratic elution program. The mobile phase consisted of acetonitrile-water $(90: 10, \mathrm{v} / \mathrm{v})$ at a flow rate of $1.0 \mathrm{~mL} / \mathrm{min}$ for $10 \mathrm{~min}$. The UV detection wavelength was set at 203 $\mathrm{nm}$. All injection volumes were $10 \mu \mathrm{L}$.

\subsection{DPPH Radical Scavenging Capacity Measurement}

The radical scavenging ability of 2,2'-diphenyl-1picrylhydrazyl (DPPH) was estimated by a method adapted from Arranz S et al [4,36]. Briefly, $1 \mathrm{ml}$ oil sample or walnut extract was vortexed for $20 \mathrm{~s}$ with $1 \mathrm{~mL}$ of the DPPH solution ( $250 \mathrm{mM}$ ) in isopropanol. The mixture was left to stand at room temperature for $40 \mathrm{~min}$ in the dark and absorbance was measured at $517 \mathrm{~nm}$. Results were expressed as Trolox equivalent antioxidant activity (TEAC) defined as micromoles of Trolox per $g$ of extract.

\subsection{ABTS Radical Scavenging Capacity Measurement}

Free radical scavenging capacity using a stable ABTS radical was performed according to Arranz $S$ et al, $[4,36]$ with some modifications. The ABTS radical solution was produced by gently mixing $2 \mathrm{~mL}$ of $7 \mathrm{mM}$ ABTS solution and $2 \mathrm{~mL}$ of $2.45 \mathrm{mM}$ potassium persulfate solution. This was allowed to stand in the dark at room temperature for 12-16 h. The ABTS radical solution was adjusted with ethanol to an absorbance of $0.8( \pm 0.02)$ at $734 \mathrm{~nm}$ before usage. Fifty $\mu \mathrm{L}$ of oil sample or walnut extract was added to $1 \mathrm{~mL}$ ABTS radical solution and allowed to react for 20 min until a stable absorbance was obtained. The results were expressed as TEAC.

\subsection{Ferric Reducing Antioxidant Potential (FRAP) Assay}

The ferric reducing power of walnut sample was determined using a modified version of the FRAP assay $[4,36]$. The working FRAP reagent was prepared by mixing 10 volumes of $300 \mathrm{mM}$ acetate buffer, $\mathrm{pH} 3.6$, with 1 volume of $10 \mathrm{mM}$ TPTZ (2,4,6-tri(2-pyridyl)-s-triazine) in $40 \mathrm{mM}$ hydrochloric acid and with 1 volume of $20 \mathrm{mM}$ ferric chloride. A standard curve was prepared using various concentrations of $\mathrm{FeSO}_{4} \times 7 \mathrm{H}_{2} \mathrm{O}$. Fifty $\mu \mathrm{L}$ of oil sample or walnut extract was added to $700 \mu \mathrm{L}$ of freshly prepared FRAP reagent. The reaction mixture was incubated for 40 min at $37^{\circ} \mathrm{C}$ in the dark. Then, the absorbance of the samples was measured at $593 \mathrm{~nm}$. A sample blank reading using acetate buffer was also taken. FRAP values were expressed as micromoles $\mathrm{Fe}^{2+} / \mathrm{g}$ of walnut extract.

\subsection{Statistical Analysis}

All the results were carried out in triplicate and the values were presented as mean and standard deviation. All statistical calculations and hierarchical cluster analysis were analyzed by SPSS statistics software (SPSS for Windows 18.0, SPSS Inc., USA).

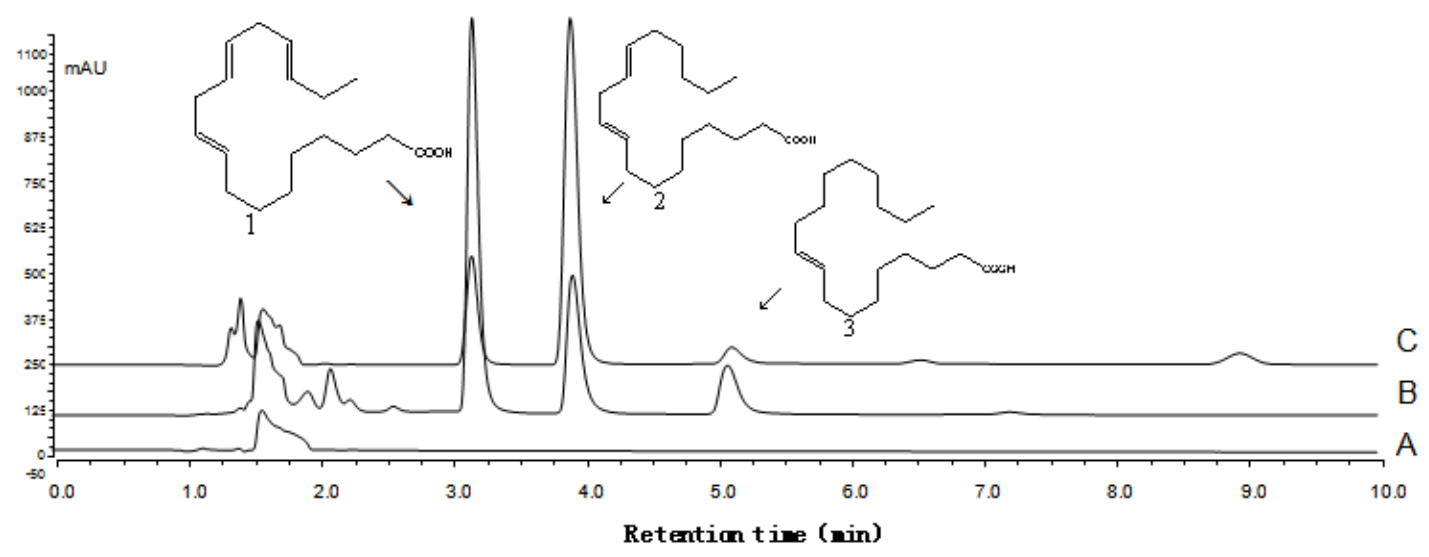

Figure 1. The chromatograms of blank solution (A), standard solution (B), sample solution (C). 1: $\alpha$-linolenic acid 2: linoleic acid 3: oleic acid 


\section{Results}

\subsection{Specificity}

The chromatograms of blank solution, standard solution and sample solution were given in Figure 1.

\subsection{Limits of Detection and Quantification}

Ethanol stock solution containing three reference compounds were diluted to a series of appropriate concentrations with the same solvent, and an aliquot of the diluted solutions were injected into HPLC for analysis. The limits of detection (LOD) and quantification (LOQ) under the present chromatographic conditions were determined at $\mathrm{S} / \mathrm{N}$ (the ratio of signal to noise) of 3 and 10 , respectively. The ratio of signal to noise was calculated using Chromeleon7.1 ChemStation software. Table 1 showed the data of LOD and LOQ for each investigated compound.

\subsection{Calibration curves}

The stock mixture standard solution was accurately injected $1,2,5,10,15,20,25 \mu \mathrm{L}$ to chromatographic instrument for the construction of calibration curves. And then the calibration curves were constructed by plotting the peak areas versus the concentration of each analyte. The results were demonstrated in Table 1.

Table 1. Calibration curves for three fatty acid

\begin{tabular}{llllll}
\hline Analytes & Regressive equation $^{\mathbf{a}}$ & $\mathbf{r}^{2}$ & Linear range $(\boldsymbol{\mu g})$ & $\mathbf{L O D}(\boldsymbol{\mu g} / \mathbf{m L})$ & $\mathbf{L O Q}(\boldsymbol{\mu g} / \mathbf{m L})$ \\
\hline $\boldsymbol{\alpha}$-linolenic acid & $\mathrm{y}=30.295 \mathrm{x}+1.2943$ & 0.9999 & $0.2828-7.070$ & 0.0283 & 0.0846 \\
linoleic acid & $\mathrm{y}=31.635 \mathrm{x}+4.7064$ & 0.9999 & $1.0340-12.950$ & 0.0296 & 0.0998 \\
oleic acid & $\mathrm{y}=6.7977 \mathrm{x}+0.8818$ & 0.9992 & $0.2558-6.395$ & 0.0512 & 0.1545 \\
\hline
\end{tabular}

${ }^{\mathrm{a}} \mathrm{y}$ : Peak area; $\mathrm{x}$ : concentration $(\mu \mathrm{g})$.

\subsection{Precision}

Intra- and inter-day variations were chosen to determine the precision of the developed assay. A certain concentration solution of three reference compounds was tested. For intra-day variability, the samples were analyzed in six replicates within 1 day. The RSD of peak areas was $0.51 \%, 2.47 \%$ and $0.43 \%$, indicating that the system was precise for quantitative analysis of $\alpha$-linolenic acid, linoleic acid and oleic acid. While for inter-day variability, the samples were examined in triplicate for three consecutive days. The RSD of peak areas was $1.41 \%$, $1.63 \%$ and $2.52 \%$, indicating that the method was precise for quantitative analysis of $\alpha$-linolenic acid, linoleic acid and oleic acid.

\subsection{Reproducibility}

Six sample solutions of the same crude oil at appropriate concentration were prepared, and then analyzed on an UltiMate -3000 HPLC instrument. The
RSD of $\alpha$-linolenic acid, linoleic acid and oleic acid contents obtained from the instrument was $2.60 \%, 0.89 \%$ and $2.86 \%$, indicating that this analytical method was able to be well reproduced to determine fatty acid in the crude oil.

\subsection{Stability}

Stabilities of standard solution were tested at room temperature. The standard solution was found to be stable in nonaqueous ethanol at room temperature within 24h, with RSD of $1.92 \%, 1.36 \%$ and $2.72 \%$, respectively.

\subsection{Accuracy}

Recovery test was used to evaluate the accuracy of this method. Accurate amounts of three fatty acids were added to approximate $0.08 \mathrm{~g}$ of crude oil, and then methoded and analyzed as described in Section 2.3. The recoveries were counted by the formula: recovery $(\%)=$ (amount found original amount)/amount added $\times 100 \%$, and RSD $(\%)=$ $(\mathrm{SD} / \mathrm{mean}) \times 100 \%$. The results were given in Table 2 .

Table 2. Recovery of the three analytes

\begin{tabular}{lllll}
\hline Analyte & Original (mg) & Added (mg) & Found (mg) & Recovery (\%) \\
\hline \multirow{3}{*}{$\boldsymbol{\alpha}$-linolenic acid } & 11.33 & 8.85 & 19.63 & 93.79 \\
& 11.29 & 11.27 & 22.11 & 96.01 \\
& 11.16 & 13.63 & 24.09 & 94.86 \\
linoleic acid & 57.51 & 45.66 & 102.99 & 99.61 \\
& 57.09 & 56.61 & 112.40 & 97.70 \\
& 56.46 & 66.81 & 121.19 & 96.89 \\
oleic acid & 17.89 & 15.26 & 32.25 & 94.10 \\
& 19.42 & 19.23 & 37.63 & 94.70 \\
& 13.54 & 16.38 & 29.21 & 95.67 \\
\hline
\end{tabular}

\section{Sample Analysis}

The developed RP-HPLC method was successfully applied for the determination of $\alpha$-linolenic acid, linoleic acid and oleic acid in walnut oil from thirteen cultivated habitats. The average contents were shown in Table 3.
Compare the methanol - water, methanol- acetic acid aqueous solution, acetonitrile - water and acetonitrile acetic acid aqueous solution. Due to the UV cut-off absorption wavelength of acetonitrile was small, so we selected to acetonitrile - water system as mobile phase. When joining a small amount of acetic acid in phase have no significant effect on the separation effect and peak shape, and for the stability of column life have certain effect. And a higher proportion of organic solvent in the mobile phase was found to decrease the retention time.

\section{Discussion}


Therefore, we selected acetonitrile - water (90:10, v/v) as mobile phase, an isocratic elution program.

All calibration curves showed good linear regression $\left(\mathrm{R}^{2}>0.9992\right)$ within test ranges. Validation studies of this method proved that this assay had good reproducibility. The results in Section 3.3 showed, the overall intra- and inter-day variations were less than $3 \%$ for all three analytes. As demonstrated in Table 2, the developed analytical method had good accuracy with the overall recovery of high than $93 \%$ for the analytes concerned. The limit of detection $(\mathrm{S} / \mathrm{N}=3)$ and the limit of quantification $(\mathrm{S} / \mathrm{N}=10)$ were less than $0.052 \mu \mathrm{g} / \mathrm{mL}$ and $0.1545 \mu \mathrm{g} / \mathrm{mL}$ on column, respectively (Table 1 ), indicating that this HPLC method was precise, accurate and sensitive enough for the quantitative evaluation of the unsaturated fatty acids in walnut oil.

It has been reported that unsaturated fatty acids were major types of fatty acids in walnut oil [22]. More importantly, the intake of foods rich in unsaturated fatty acids, such as those contained in most vegetable fats and oils and oily fish, was associated with improving lipid profiles, a lower potency of intermediate biomarkers of atherosclerosis and lesser incidence of cardiovascular diseases [5,27]. So we adopted three unsaturated fatty acids as representative markers to establish a quality control method.

The newly established HPLC-UV method was applied to analyze three analytes in thirteen cultivated habitats (Table 3). It was found that there were remarkable differences of three unsaturated fatty acids contents between different plant sources of walnut. All three unsaturated fatty acids analyzed could be detected in thirteen cultivated habitats. Among all three unsaturated fatty acids interested, linoleic acid was determined as the major unsaturated fatty acids with content of 47.10-56.58 $\mathrm{g} / 100 \mathrm{~g}$. The results herein reported suggest that, besides genetic factors, the unsaturated fatty acids contents can be strongly influenced by environmental factors.

Table 3. The average contents ( $\mathrm{g} / \mathbf{1 0 0 g})$ of different habitats $(\mathrm{n}=3)$

\begin{tabular}{|c|c|c|c|c|}
\hline No & Habitats & $\alpha$-linolenic acid & linoleic acid & oleic acid \\
\hline S1 & Shennongjia & 13.56 & 47.10 & 18.63 \\
\hline S2 & Mengyinxian & 12.11 & 53.96 & 12.37 \\
\hline S3 & Yunnan & 11.11 & 54.55 & 19.45 \\
\hline S4 & akesu & 13.64 & 51.67 & 12.49 \\
\hline S5 & Longxian & 11.45 & 50.15 & 27.27 \\
\hline S6 & Qixingguan & 12.28 & 54.39 & 24.53 \\
\hline S7 & Jiaochengxian & 11.67 & 48.57 & 21.17 \\
\hline S8 & Zanhuangxian & 12.62 & 50.72 & 22.87 \\
\hline s9 & Zhengningxian & 11.33 & 54.67 & 15.24 \\
\hline S10 & Huixian & 11.66 & 52.14 & 10.69 \\
\hline S11 & Shunqingxian & 13.25 & 50.16 & 11.81 \\
\hline S12 & Hezhangxian & 10.55 & 56.58 & 23.60 \\
\hline S13 & Weiningxian & 7.90 & 49.74 & 24.36 \\
\hline
\end{tabular}

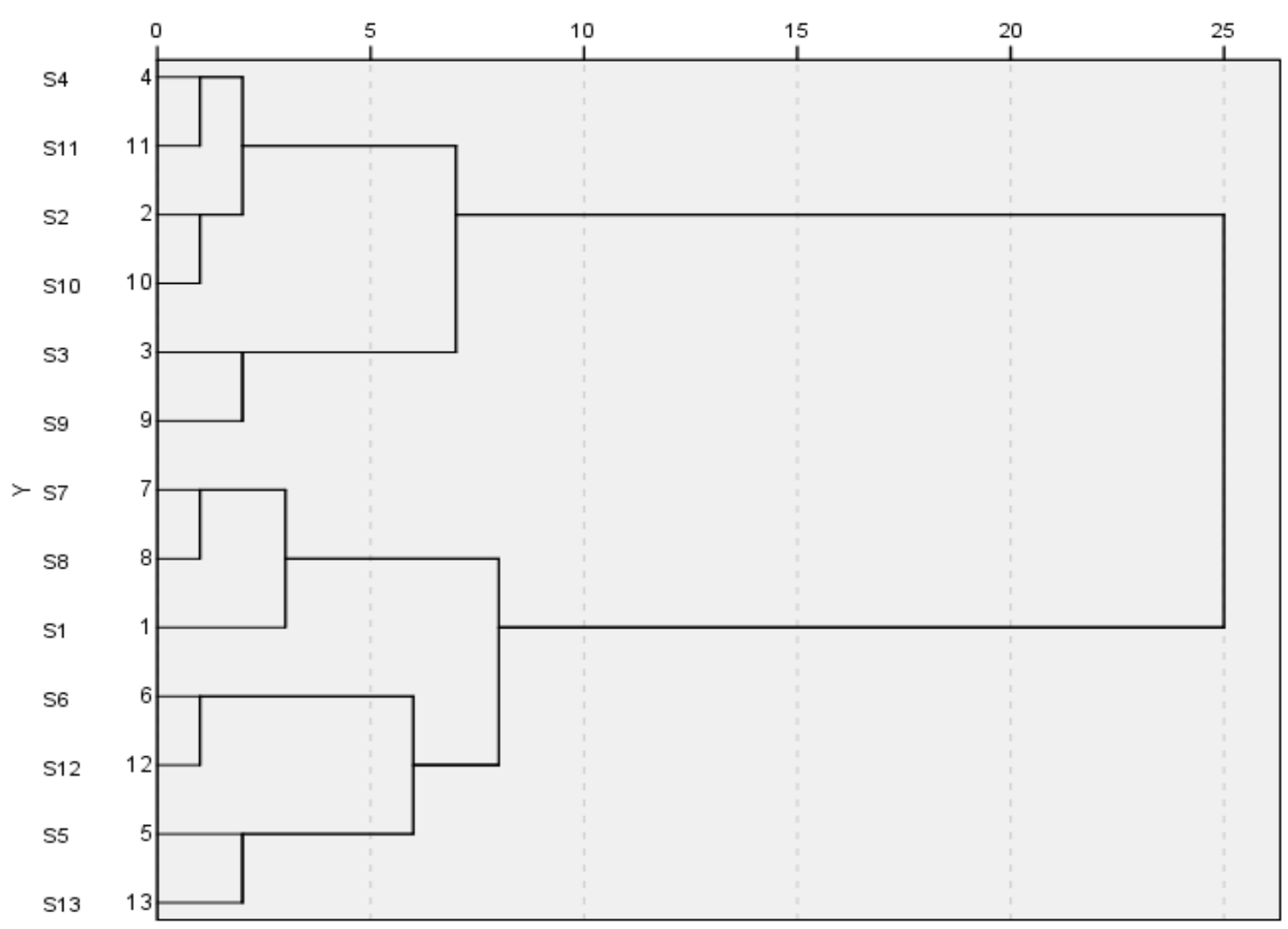

Figure 2 Hierarchical cluster analysis of walnut oil samples 
To further visualize the relationships of various walnut oil samples from different origins, hierarchical cluster analysis (HCA) was performed on the peak areas of analytes. Hierarchical clustering analysis (HCA), a multivariate analysis technique, is used to discriminate different samples. The result of HCA was shown in Figure 2. It was obvious that the samples could be classified into four clusters: S4, S11, S2, and S10, in cluster 1, S3 and S9 in cluster 2, S8, S7 and S1 in cluster 3 and S6, S12, S5 and S13 in cluster 4. All of the samples in cluster 4 collected from Guizhou Province except for S5 (Longxian, Shanxi Province). The results were showed that HCA could provide an effectively distinguish the different habitats of walnut oil.

Table 4 shows the TEAC of the walnut kernel extract and walnut oil. The walnut oil had a lower antioxidant activity than the walnut, which may be related to the walnut kernel was rich in antioxidative polyphenols [15]. Antioxidant active ingredients are known to have protective effects against chronic diseases [8]. Antioxidant ingredients were found in the walnut oil, which have been found to possess antioxidant activity. It can be interpreted that the walnut oil may be utilized as sources of dietary antioxidants.

Table 4. The antioxidant activity of the walnut oil

\begin{tabular}{llll}
\hline Sample & DPPH $(\boldsymbol{\mu M}$ TEAC/g) & ABTS $(\boldsymbol{\mu M}$ TEAC/g) & FRAP $(\boldsymbol{\mu M}$ TEAC/g) \\
\hline Walnut oil $(\mathbf{1 0 0} \mathbf{m g} / \mathbf{m L})$ & $0.47 \pm 0.01$ & $1.24 \pm 0.15$ & $1.86 \pm 0.11$ \\
Walnut extract $(\mathbf{5 0} \boldsymbol{\mu g} / \mathbf{m L})$ & $1026.19 \pm 52.95$ & $2754.30 \pm 42.79$ & $4180.61 \pm 41.99$ \\
\hline
\end{tabular}

\section{Conclusions}

Generally, fatty acids were determined by GC-MS as the esters after derivatization, which had the drawbacks such as prolonged analysis time, additional cost for derivatization and so on. This research established a method with simplified the sample preparation, which was simple rapid and accurate, can be used to simultaneously determination the unsaturated fatty acids in walnut oil without derivatization. It was helpful to control the quality of the walnut oil. The walnut oil was found to have antioxidant activity measured by Trolox equivalent antioxidant capacity assay. It can be concluded that the walnut oil may be utilized as a source of phytonutrients.

\section{Acknowledgments}

The authors gratefully acknowledge the financial support of the present work by the National Science and Technology Support Plan Project of China (No. 2011BAC09B01-02-04), the Characteristic Key Laboratory Construction Project of Guizhou Province Education Department (No. KY [2012]005), the Social Development Project of Guizhou Science and Technology Department (No. SY [2012]3066), High Technology Project of Guizhou Province Development and Reform Commission (No. [2013]2068), and Science and Technology Plan Project of Guizhou Province (No. ZY [2011]3013).

\section{References}

[1] Amaral JS, Casal S, Pereira JA, Seabra RM, Oliveira BPP, "Determination of Sterol and Fatty Acid Compositions, Oxidative Stability, and Nutritional Value of Six Walnut (Juglans regia L.) Cultivars Grown in Portugal," J Agric food chem, 51(26). 7698702. 2003.

[2] Simopoulos AP S, "The importance of the ratio of omega-6/ omega-3 essential fatty acids," Biomed Pharmacother, 56. 365-79. 2002.

[3] Aparicio R, Aparicio-Ruiz R, "Authentication of vegetable oils by chromatographic techniques," J Chromatogr A, 881. 93-104. 2000.

[4] Arranz S, Pérez-Jiménez J, Saura-Calixto F, “Antioxidant capacity of walnut (Juglans regia L.): contribution of oil and defatted matter,” Eur Food Res Technol, 227(2). 425-31. 2007.
[5] Banel DK, Hu FB, "Effects of walnut consumption on blood lipids and other cardiovascular risk factors: a meta-analysis and systematic review,” Am J Clin Tutr, 90(1). 56-63. 2009.

[6] Beyhan OE, Kaya I, Sen SM, Dogan M, "Fatty Acids Composition of Walnut (Juglans regia L.) Types Selected in Darende,” Turk J Agric For, 19. 299-302. 1995.

[7] Bravi E, Perretti G, Montanari L, "Fatty acids by highperformance liquid chromatography and evaporative lightscattering detector,” J Chromatogr A, 1134(1-2). 210-4. 2006.

[8] Cai Yi-zhong, Luo Qiong, Sun Mei, Harold Corke, “Antioxidant activity and phenolic compounds of 112 traditional Chinese medicinal plants associated with anticancer," Life Sciences, 74. 2157-2184. 2004

[9] Calvo P, Lozano M, Espinosa-Mansilla A, González-Gómez D, "In-vitro evaluation of the availability of $\varpi-3$ and $\varpi-6$ fatty acids and tocopherols from microencapsulated walnut oil," Food Res Int, 48(1). 316-21. 2012.

[10] Carey AN, Fisher DR, Joseph JA, Shukitt-Hale B, "The ability of walnut extract and fatty acids to protect against the deleterious effects of oxidative stress and inflammation in hippocampal cells," Nutr Neurosci, 16(1). 13-20. 2013.

[11] Crews C, Hough P, Godward J, Brereton P, Lees M, Guiet S, Winkelmann W, "Study of the main constituents of some authentic walnut oils,” J Agric Food Chem, 53(12). 4853-60. 2005.

[12] Cunha S, Oliveira M, "Discrimination of vegetable oils by triacylglycerols evaluation of profile using HPLC/ELSD," Food Chem, 95(3). 518-24. 2006.

[13] Dierkes G, Krieger S, Duck R, Bongartz A, Schmitz OJ, Hayen H, "High-performance liquid chromatography-mass spectrometry profiling of phenolic compounds for evaluation of olive oil bitterness and pungency,” J Agric Food Chem, 60(31). 7597-606. 2012.

[14] Ding Y, Tang X, "Determination of fatty acids in Fructus Bruceae oil by precolumn derivation HPLC," Chin Tradit Herbal Drugs, 35(9). 988-91. 2004.

[15] Fukuda T, Ito H, Yoshida T, "Antioxidative polyphenols from walnuts (Juglans regia L.),” Phytochemistry, 63(7). 795-801. 2003.

[16] Garcia JM, Agar IT, Streif J, "Lipid Characterization in Kernels from Different Walnut Cultivars," Turk J Agric For, 18. 195-8. 1994.

[17] Gharibzahedi SMT, Mousavi SM, Hamedi M, Rezaei K, Khodaiyan F, "Evaluation of physicochemical properties and antioxidant activities of Persian walnut oil obtained by several extraction methods," Ind Crop Prod, 45.133-40. 2013.

[18] Greve C, McGranahan G, Hasey J, Snyder R, Kelly K, Goldhamerer D, Labavitch J, "Variation in Polyunsaturated Fatty Acid Composition of Persian Walnut," J Soc Hort Sci, 117. 51822. 1992.

[19] Khattab RY, Zeitoun MA, "Quality evaluation of flaxseed oil obtained by different extraction techniques," LWT - Food Sci Technol, 53(1). 338-45. 2013.

[20] Li XJ, Wang W, Luo M, Li CY, Zu YG, Mu PS, Fu YJ, “Solventfree microwave extraction of essential oil from Dryopteris fragrans and evaluation of antioxidant activity," Food Chem, 133(2). 437-44. 2012.

[21] Marangoni F, Colombo C, Martiello A, Poli A, Paoletti R, Galli C. "Levels of the n-3 fatty acid eicosapentaenoic acid in addition to 
those of alpha linolenic acid are significantly raised in blood lipids by the intake of four walnuts a day in humans," Nutr Metab Cardiovasc Dis, 17(6). 457-61. 2007.

[22] Martínez M, Barrionuevo G, Nepote V, Grosso N, Maestri D, "Sensory characterisation and oxidative stability of walnut oil," Int J Food Sci Tech, 46(6). 1276-81. 2011.

[23] Miraliakbari H SF, "Oxidative stability of tree nut oils," J Agric Food chem, 56(12). 4751-9. 2008.

[24] Nagel JM, Brinkoetter M, Magkos F, Liu X, Chamberland JP, Shah S, Zhou J, Blackburn G, Mantzoros CS, "Dietary walnuts inhibit colorectal cancer growth in mice by suppressing angiogenesis," Nutrition, 28(1). 67-75. 2012.

[25] Pereira JA, Oliveira I, Sousa A, Ferreira IC, Bento A, Estevinho L, "Bioactive properties and chemical composition of six walnut (Juglans regia L.) cultivars,” Food chem Toxicol, 46(6). 2103-11. 2008.

[26] Prasad RBN, Walnuts and Pecans, in Encyclopaedia of Food Science. Food, Technology and Nutrition Academic Press, London, 1994, pp 4828-4831.

[27] Ros E, Mataix J, "Fatty acid composition of nuts - implications for cardiovascular health,” British J Nutr, 96(S2). S29. 2006.

[28] Tian Y, Xu Z, Zheng B, Martin Lo Y, "Optimization of ultrasonicassisted extraction of pomegranate (Punica granatum L.) seed oil,” Ultrason Sonochem, 20(1). 202-8. 2013.
[29] Ulberth F BM, "Authenticity of fats and oils," Eur J Lipid Sci Technol, 102. 687-94. 2000.

[30] Vanschoonbeek K dMM, Heemskerk JWM, "Fish oil consumption and reduction of arterial disease,” J Nutr, 133. 657-60. 2003.

[31] Wang SH, Shi PY, Feng FQ, "Study on saponification technology of Hippophae rhamnoides L. seed oil," Sci Technol Food Ind, 30(06). 212-7. 2009.

[32] Wang XL, YMM, Chi N, Tang X, "Determination of the content of fatty acids in Hippophae rhamnoides L. seed oil by HPLC with precolumn derivatization," J Shenyang Pharm Univ, 27(10). 767812. 2010.

[33] Zeb A, "Triacylglycerols composition, oxidation and oxidation compounds in camellia oil using liquid chromatography-mass spectrometry,” Chem Phys Lipids, 165(5). 608-14. 2012.

[34] Ros E, Mataix J, "Fatty acid composition of nuts - implications for cardiovascular health,” British Journal of Nutrition, 96(S2). S29. 2006.

[35] Wang S-H, Feng F-Q, "Study on saponification technology of Hippophae rhamnoides L. seed oil,” Sci Technol Food Ind, 30(06). 212-7. 2009.

[36] Lin Li, Hyeonmi Ham, Jeehye Sung, Younghwa Kim, Heon-Sang, Jeongand Junsoo Lee, "Antioxidant Activities of Methanolic Extracts from Four Different Rose Cultivars,” Journal of Food and Nutrition Research, 2(02). 69-73. 2014. 